INPLASY

PROTOCOL

To cite: Wang. Effect of

Resistance Training on Energy Intake and Appetite Regulation among Overweight and Obese People: A Systematic Review. Inplasy protocol 202210090. doi:

10.37766/inplasy2022.1.0090

Received: 17 January 2022

Published: 17 January 2022

Corresponding author:

Qiang Wang

w545537572@sina.com

Author Affiliation:

Universiti Putra Malaysia

Support: No.

Review Stage at time of this submission: Completed but not published.

Conflicts of interest:

None declared.

\section{Effect of Resistance Training on Energy Intake and Appetite Regulation among Overweight and Obese People: A Systematic Review}

Wang, Q1.

Review question / Objective: This systematic review analyzes the effect of resistance training on energy intake and appetite regulation among overweight or obese people. Although the benefits and effects of resistance training have been analyzed in the literature, there is minimal direct scientific evidence on the effect of resistance training on energy intake and appetite regulation among overweight or obese people. Therefore, it is essential to analyze related scientific evidence on the potential benefits and risks of resistance training before impacting energy intake and appetite regulation. Therefore, this systematic review aimed to provide a comprehensive critical commentary on the current literature on the effect of resistance training on energy intake and appetite regulation among overweight and obese people and offer recommendations for the best practice.

INPLASY registration number: This protocol was registered with the International Platform of Registered Systematic Review and Meta-Analysis Protocols (INPLASY) on 17 January 2022 and was last updated on 17 January 2022 (registration number INPLASY202210090).

\section{INTRODUCTION}

Review question / Objective: This systematic review analyzes the effect of resistance training on energy intake and appetite regulation among overweight or obese people. Although the benefits and effects of resistance training have been analyzed in the literature, there is minimal direct scientific evidence on the effect of resistance training on energy intake and appetite regulation among overweight or obese people. Therefore, it is essential to analyze related scientific evidence on the potential benefits and risks of resistance training before impacting energy intake and appetite regulation. Therefore, this systematic review aimed to provide a 
comprehensive critical commentary on the current literature on the effect of resistance training on energy intake and appetite regulation among overweight and obese people and offer recommendations for the best practice.

Rationale: Obesity can be defined as the result of an energy imbalance. Increased energy intake through food intake is not conducive to energy consumption. Energy consumption consists of three parts: primary metabolism, postprandial thermogenesis, and physical activity. Physical activity is the most variable, and overweight or obese people are known to have less physical activity, while their food intake usually increases. The complex energy balance regulation system is affected by physical exercise. Although some studies have shown that it is relevant to the hormonal stimulation related to food intake, other studies have shown that exercise can bring satiety. Studies have shown that although short-term exercise can increase energy consumption, longterm exercise can also affect appetite and total energy consumption. Scholars have been encouraging overweight and obese people to eat less and be more active and studying effective training methods to lose their weight by reducing their energy intake and increase their perceived fullness. Among them, resistance training may be the unique exercise way that affects diet intake and non-RT physical activity. Scholars has already reported that initiation of a RT intervention (e.g., Initiation Phase of the Resist Diabetes trial) was associated with a reduction in reported energy, carbohydrate, total sugar, fruit and vegetable, and sweets and dessert intake over three months. Although children and adolescents have traditionally been encouraged to participate in aerobic exercise, resistance training has proved to be a safe and effective conditioning method for all young people, regardless of their size. Several studies have reported that resistance training programs can lead to positive changes in obese children and adolescents. Some studies have pointed out that compared with aerobic exercise alone, the combined training of aerobic and resistance training groups can better reduce the diet intake of obese adolescents after six months. Food intake comprises 3 phases. The first, the ingestive phase, is characterized by hunger. The second, the prandial phase, corresponds to food intake when satiation occurs. The last phase, the postprandial phase, is characterized by satiety. The traditional glucostatic theory maintains that food consumption is activated because of a decrease in blood glucose. However, the regulation of food intake is part of a complex system that involves hormonal signaling throughout the body. These hormones can be divided into two categories, anorectic (appetite suppressing) and orexigenic (appetitestimulating). The main hormones regulating appetite are insulin, leptin, NPY, active ghrelin, pancreatic polypeptide, peptide YY, adiponectin which scholars have already researched the effect of resistance training on them. Each hormone has its corresponding mechanism of action. In addition, due to the leptin resistance mechanism, some scholars also study the effect of resistance training on the HOMAIR index through the HOMA method. Existing studies show that resistance training is likely to reduce the appetite of inactive overweight or obese people by suppressing orexigenic hormones and stimulating the anorexigenic hormones. Although the benefits and effects of either resistance training or energy intake and appetite regulation have been analyzed in the literature, the systematic scientific evidence on the effects of resistance training on energy intake and appetite regulation among overweight or obese people is minimal. Therefore, it is crucial to analyze the scientific evidence on the potential benefits and risks of resistance training before conducting its effects on energy intake and appetite regulation. Therefore, the purpose of this systematic review is to assess the effectiveness of published research on the effect of resistance training on energy intake or appetite regulation among overweight or obese people. 
Condition being studied: Field of sports science and physiology.

\section{METHODS}

Search strategy: Firstly, it is searched in EBSCOHOST, Scopus, Pub Med, and Web of Science systems. Enter the keywords and synonyms "resistance training" OR "resistance exercise" OR "strength training" OR "strength exercise" OR "weight training" OR "weight lifting" AND "appetite" OR "hunger" OR" food intake" OR "food consumption" OR "energy intake" OR "intake energy" OR "Calorie intake" OR "intake calorie" AND "obesity" OR "overweight" OR "fat" OR "obese" OR "unhealthy weight" OR" high BMI" is searched for the effect of resistance training on energy intake and appetite among overweight or obese people. Searches were limited to papers published in English and from 1 January 1980 to 17 January 2022. Secondly, starting to use EBSCOHOST, Scopus, Pub Med, Web of Science, and article retrieval systems to input keywords and analyze the title of the required articles. The title of the article is analyzed. The article's title should include resistance training, energy intake or appetite, and being overweight or obese. Then, the abstract of the selected article is analyzed. Full-text data extraction as follows: i. the author, keywords (resistance training, energy intake or appetite, and overweight or obese). ii. this article must experiment (group control experiment, a randomized controlled trial, a set of experiments), iii. intervention is resistance training, the sample is overweight and obese people, the result and conclusion must be above one of energy intake, perceive appetite or appetite-related hormones. Universiti Putra Malaysia's librarians also assisted this study. A systematic literature search was carried out using EBSCOhost, Scopus, Pub Med, Web of Science database, and additional records were identified through other sources - Google scholar and references which included 277 articles and 432 subjects in total. We reviewed the bibliography of qualified articles and related reviews. Randomized, compared, and single-group trials were included to evaluate the effects of resistance training on energy intake and appetite regulation among overweight or obese people, and qualified review studies were synthesized according to PRISMA guidelines. A checklist of three authors evaluated the quality of the study.

Participant or population: Overweight or obese healthy and sedentary people, not been on a diet recently, must be more than 10 subjects.

Intervention: Must have single Resistance training group, more than 4 weeks without energy intake restriction or regular extra supply, and intervention project accord with the energy intake and appetite regulation.

Comparator: The study involved two or more groups and single-group trials. Outcomes: Results include energy intake, perceived appetite, or appetite-related hormones affected by resistance training.

Study designs to be included: Experiment research including RCT and non-RCT.

Eligibility criteria: For a study to be eligible, each of the following inclusion criteria weremet: $i$. the subjects of study must be overweight or obese people previously. The reason why selecting this kind of people is that regular exercise training on appetite regulation may be of most relevance to this population. ii. the independent variable is resistance training and was defined as any one of high, medium, low intensity, or other resistance training, but it needs to be a separate resistance training. iii. Study method included in the article is the experimental research (randomized controlled trials, comparative trials, singlegroup trials) in resistance training for intervention to control energy intake or appetite regulation as the primary goal.iv. the intervention period cannot be less than four weeks. The criteria were adopted because neuromuscular adaptation was not observed in individuals with resistance 
training in less than four weeks. v. the number of intervened people may not be less than ten people. Otherwise, the sample will lack adequate representation of the whole population, so it is not easy to ensure the accuracy and reliability of the calculation results.vi. intervention project will accord with the requirement of energy intake or appetite regulation, which are energy intake, appetite perception, and appetite-related hormones. Physiological research is an exact work, and we can ensure the accuracy and effectiveness of the final data only in this way.vii. the influence of resistance training on energy intake and appetite among overweight or obese people is evaluated, and at least one of them can be divided into results.viii. full-text formal articles written in English and published in a peer-reviewed journal.2.3.2 Studies were excluded if any of the following criteria applied:i. the participants should have not any disease or taking any medicines.ii. the subjects should lack active exercise training and be not on a diet recently in the last three months. iii. during the intervention, it should not have any energy intake restriction or extra compulsory supplements.iv. the resistance training should not just be embedded as part of whole training in the resistance training group, such as both resistance training and aerobic training was carried out in the intervention group at the same time. $v$. the resistance training intervention was poorly controlled and reported.

Information sources: A systematic literature search was carried out using EBSCOhost, Scopus, Pub Med, Web of Science database, and additional records were identified through other sources Google scholar and references which included 277 articles and 432 subjects in total.

Main outcome(s): In half of the relevant articles, the energy intake of obese or overweight people affected by resistance training is significantly reduced. Methods to record the food intake were 3-day food records. All energy intake analysis methods use different nutrition analysis software. Most studies show that resistance training can significantly change the glucose index. In the groups with significant differences, most of the glucose index decreased, and most resistance training intensity was high. About leptin, Most studies have shown that resistance training can significantly reduce leptin concentration, and the leptin of the high-intensity group was significantly lower than other intensity groups. About HOMAIR, One article reported a significantly lower index after the experiment among all low, moderate, and high intensity three groups separately, and the index of the high-intensity group was significantly lower than the other groups. About NPY, an article on low and high-intensity resistance training showed a significant reduction of NPY individually. The medium-intensity resistance training showed a significant increase of adiponectin in one article about adiponectin.

\section{Additional outcome(s): None.}

Quality assessment / Risk of bias analysis: An assessment of the study quality according to the PEDro list is presented. The data showed that the included studies were of fair quality, and none met all the PEDro list quality criteria. All studies specified their eligibility criteria, group similar at baseline, between-group comparisons, point measure and variability. No studies have reported allocation concealment, blind subject, blind therapist, blind assessor, intention to treat analysis, except for six studies that reported random allocation and three studies reported follow-up. However, blind subject, blind therapist, the blind assessor was difficult in participants and assessors since the included studies were exercise training interventions. Consequently, higher quality and evidence levels of studies should be conducted in the future. Overall, all studies were all scored a 4 or 5 on the PEDro scale. The total PEDro scores of 4-5 are considered 'fair', so the quality of all eight articles is acceptable. 
Strategy of data synthesis: In order to identify relevant studies, the search results are imported into a published software for systematic review, and two independent reviewers perform a blind review process. In the first screening stage, two independent reviewers ( $\mathrm{Li}$ Long and Wang Qiang) screened the titles and abstracts of all identified literature. After evaluating all other titles and abstracted papers using our predetermined inclusion and exclusion criteria, the irrelevant papers were removed from the database. Suppose the title and summary do not provide enough information. The full text is evaluated. The full text of potentially qualified literature was reviewed in the second screening stage. If there is any disagreement, first, try to resolve differences by consensus; if problems still cannot be resolved finally, a third reviewer (Cao Shu Dian) will be consulted until a consensus is reached. After the evaluation of the full text. Eight pieces of literature met our inclusion criteria and were therefore included in the systematic evaluation. The search produced 277 papers. By manual selection, eliminating repetitive articles $(n=85)$. Following the removal of duplicates, publications were filtered by reading the title and abstract, resulting in a further 154 papers being excluded for the following reasons: not written in English $(n=8)$, not related to the topic $(\mathrm{N}=99)$, review, conference paper note undefined, book, document school and program $(\mathrm{N}=11)$, No full text $(\mathrm{N}=17)$, the intervention has energy intake restriction or regular extra supply $(n=19)$. Thirty-eight potentially relevant papers were left which were considered in detail for appropriateness, resulting in a further 30 papers being excluded for the following reasons: subjects are not overweight or obese humans, or number is not enough $(n=8)$, subjects are patient $(n=6)$, subjects went on a diet or were not sedentary previously $(n=2)$, not single resistance training $(n=4)$, not involved intervention $(n=3)$, intervention not compliance with principles or poor reported $(n=4)$, not energy intake, perceive appetite or appetite-related hormones $(n=3)$.
Subgroup analysis: 3.3 Participant Characteristics; 3.4 Interventions Characteristics; 3.5 Outcome; 3.5.1 Effect of Resistance Training on Energy Intake Among Overweight or Obese People. 3.5.2 Effect of Resistance Training on Perceive Appetite Among Overweight or Obese People; 3.5.3 Effect of Resistance Training on Appetite Related Hormones, glucose and HOMA-IR Among Overweight or Obese People. 3.5.3. (A) Glucose: 3.5.3. (B) Insulin; 3.5.3. (C) Leptin: 3.5.3. (D) Insulin resistance (HOMA-IR): 3.5.3. (E) Neuropeptide Y (NPY): 3.5.3. (F) Ghrelin: 3.5.3. (G) Pancreatic polypeptide (PP): 3.5.3. (H) Peptide YY (PYY): 3.5.3. (I) Adiponectin.

Sensitivity analysis: 2.3.1 For a study to be eligible, each of the following inclusion criteria were met: $i$. the subjects of study must be overweight or obese people previously. The reason why selecting this kind of people is that regular exercise training on appetite regulation may be of most relevance to this population. ii. the independent variable is resistance training and was defined as any one of high, medium, low intensity, or other resistance training, but it needs to be a separate resistance training. iii. Study method included in the article is the experimental research (randomized controlled trials, comparative trials, single-group trials) in resistance training for intervention to control energy intake or appetite regulation as the primary goal. iv. the intervention period cannot be less than four weeks. The criteria were adopted because neuromuscular adaptation was not observed in individuals with resistance training in less than four weeks. v. the number of intervened people may not be less than ten people. Otherwise, the sample will lack adequate representation of the whole population, so it is not easy to ensure the accuracy and reliability of the calculation results. vi. intervention project will accord with the requirement of energy intake or appetite regulation, which are energy intake, appetite perception, and appetite-related hormones. Physiological research is an exact work, and we can 
ensure the accuracy and effectiveness of the final data only in this way. vii. the influence of resistance training on energy intake and appetite among overweight or obese people is evaluated, and at least one of them can be divided into results. viii. full-text formal articles written in English and published in a peer-reviewed journal. 2.3.2 Studies were excluded if any of the following criteria applied: i. the participants should have not any disease or taking any medicines. ii. the subjects should lack active exercise training and be not on a diet recently in the last three months. iii. during the intervention, it should not have any energy intake restriction or extra compulsory supplements. iv. the resistance training should not just be embedded as part of whole training in the resistance training group, such as both resistance training and aerobic training was carried out in the intervention group at the same time. $v$. the resistance training intervention was poorly controlled and reported.

Language: English.

Country(ies) involved: Malaysia, China.

Keywords: Resistance training, energy intake, appetite, overweight, obese.

Contributions of each author:

Author 1 - Qiang Wang. 